\title{
Divisibility of twisted Alexander polynomials and fibered knots
}

\author{
Teruaki Kitano AND TAKayuki Morifuji
}

\begin{abstract}
We prove that Wada's twisted Alexander polynomial of a knot group associated to any nonabelian $S L(2, \mathbb{F})$-representation is a polynomial. As a corollary, we show that it is always a monic polynomial of degree $4 g-2$ for a fibered knot of genus $g$.
\end{abstract}

Mathematics Subject Classification (2000): 57M25 (primary); 57M05 (secondary).

\section{Introduction}

In the early ' 90 s, the theory of twisted Alexander polynomials was introduced by Lin [11] and Wada [17] independently (the article [11] appeared first in 1990 as a Columbia University preprint). Lin defined the twisted Alexander polynomial for knots using regular Seifert surfaces. On the other hand, Wada defined the twisted Alexander polynomial for finitely presentable groups, which include the link groups. Let $\Gamma$ be a finitely presentable group with a surjective homomorphism from $\Gamma$ to a free abelian group. For each linear representation of $\Gamma$, its twisted Alexander polynomial of $\Gamma$ is defined to be a rational function with some variables. This invariant in Wada's framework is sufficiently useful and computable. For instance, the Kinoshita-Terasaka knot can be distinguished from the Conway knot by this invariant. Another advantage of Wada's definition of the twisted Alexander polynomial is that it naturally coincides with the Reidemeister torsion in the case of knot groups [8].

However, the condition that the twisted Alexander polynomial becomes really a polynomial, it is not so clear. The purpose of the present paper is to give a sufficient condition that the twisted Alexander polynomial in Wada's sense becomes a polynomial. Namely, we prove the following main theorem.

The first author is supported in part by the Grant-in-Aid for Scientific Research (No. 14740037), The Ministry of Education, Culture, Sports, Science and Technology, Japan.

Pervenuto alla Redazione l'11 ottobre 2004 e in forma definitiva il 25 gennaio 2005. 
Theorem 1.1. The twisted Alexander polynomial of a knot group for any nonabelian representation into $S L(2, \mathbb{F})$ over a field $\mathbb{F}$ is always a polynomial.

As a corollary, we can show that if $K$ is a fibered knot of genus $g$, then its twisted Alexander polynomial becomes a monic polynomial of degree $4 g-2$ for any nonabelian $S L(2, \mathbb{F})$-representation (see also [3]). This result can be regarded as a generalization of the classical result, due to Neuwirth [14], which asserts the Alexander polynomial of any fibered knot is given by a monic polynomial of degree $2 g$.

After Lin and Wada, Jiang and Wang defined in [5] an invariant for 3manifolds from the viewpoint of twisting a given invariant by a representation. In [6], Kirk and Livingston gave a wide generalization of the work mentioned above and cleared the relationship between the former results and their invariants. As for other applications of the twisted Alexander polynomial, see also [4, 7, $9,12,13]$ and [16] for instance.

AcKnowledgements. The authors would like to thank Andrei Pajitnov for his interest in this work. The authors also would like to thank Masaaki Wada and Hiroshi Goda for their careful reading of an earlier version of this paper.

\section{Twisted Alexander polynomial}

For a knot group $G(K)=\pi_{1} E(K)$, namely the fundamental group of the exterior $E(K)=S^{3}-N(K)$ of a knot $K$ in the 3-sphere $S^{3}$, where $N(K)$ denotes an open tubular neighborhood of $K$, we choose and fix a Wirtinger presentation

$$
P(G(K))=\left\langle x_{1}, \ldots, x_{u} \mid r_{1}, \ldots, r_{u-1}\right\rangle .
$$

Then the abelianization homomorphism

$$
\alpha: G(K) \rightarrow H_{1}(E(K), \mathbb{Z}) \cong \mathbb{Z}=\langle t\rangle
$$

is given by

$$
\alpha\left(x_{1}\right)=\ldots=\alpha\left(x_{u}\right)=t .
$$

Here we specify a generator $t$ of $H_{1}(E(K), \mathbb{Z})$ and denote the sum in $\mathbb{Z}$ multiplicatively. In this paper, we only consider a representation $\rho: G(K) \rightarrow$ $S L(2, \mathbb{F})$, where $\mathbb{F}$ stands for a field.

These maps naturally induce two ring homomorphisms $\tilde{\rho}: \mathbb{Z}[G(K)] \rightarrow$ $M(2, \mathbb{F})$ and $\tilde{\alpha}: \mathbb{Z}[G(K)] \rightarrow \mathbb{Z}\left[t, t^{-1}\right]$, where $\mathbb{Z}[G(K)]$ is the group ring of $G(K)$ and $M(2, \mathbb{F})$ is the matrix algebra of degree 2 over $\mathbb{F}$. Then $\tilde{\rho} \otimes \tilde{\alpha}$ defines a ring homomorphism

$$
\mathbb{Z}[G(K)] \rightarrow M\left(2, \mathbb{F}\left[t, t^{-1}\right]\right)
$$


Let $F_{u}$ denote the free group on generators $x_{1}, \ldots, x_{u}$ and

$$
\Phi: \mathbb{Z}\left[F_{u}\right] \rightarrow M\left(2, \mathbb{F}\left[t, t^{-1}\right]\right)
$$

the composition of the surjection $\mathbb{Z}\left[F_{u}\right] \rightarrow \mathbb{Z}[G(K)]$ induced by the presentation $P(G(K))$ and the map $\tilde{\rho} \otimes \tilde{\alpha}: \mathbb{Z}[G(K)] \rightarrow M\left(2, \mathbb{F}\left[t, t^{-1}\right]\right)$.

Let us consider the $(u-1) \times u$ matrix $M$ whose $(i, j)$-component is the $2 \times 2$ matrix

$$
\Phi\left(\frac{\partial r_{i}}{\partial x_{j}}\right) \in M\left(2, \mathbb{F}\left[t, t^{-1}\right]\right),
$$

where $\partial / \partial x$ denotes the free differential calculus. This matrix $M$ is called the Alexander matrix of the presentation $P(G(K))$ associated to the representation $\rho$.

For $1 \leq j \leq u$, let us denote by $M_{j}$ the $(u-1) \times(u-1)$ matrix obtained from $M$ by removing the $j$ th column. We regard $M_{j}$ as a $2(u-1) \times 2(u-1)$ matrix with coefficients in $\mathbb{F}\left[t, t^{-1}\right]$.

Then Wada's twisted Alexander polynomial of a knot $K$ for a representation $\rho: G(K) \rightarrow S L(2, \mathbb{F})$ is defined to be a rational function

$$
\Delta_{K, \rho}(t)=\frac{\operatorname{det} M_{j}}{\operatorname{det} \Phi\left(x_{j}-1\right)}
$$

and moreover well-defined up to a factor $t^{2 k}(k \in \mathbb{Z})$. See [17] and [3] for details.

As a criterion for the twisted Alexander polynomial to be a polynomial, Wada shows in [17] the following:

Proposition 2.1. Let $\rho: G(K) \rightarrow S L(2, \mathbb{F})$ be a representation of a knot group $G(K)$ so that there exists an element $\gamma$ of the commutator subgroup $[G(K), G(K)]$, whose image $\rho(\gamma)$ does not have the eigenvalue 1 . Then $\operatorname{det} M_{j}$ is divisible by $\operatorname{det} \Phi\left(x_{j}-1\right)$.

Remark 2.2. In [17] Proposition 9, Wada also shows that the twisted Alexander polynomial of any link $L$ with two or more components becomes always a Laurent polynomial.

\section{Divisibility of twisted Alexander polynomials}

As for divisibility of the twisted Alexander polynomial, we obtain the following theorem.

Theorem 3.1. Let $\rho: G(K) \rightarrow S L(2, \mathbb{F})$ be a nonabelian representation of a knot group $G(K)=\pi_{1} E(K)$. Then the twisted Alexander polynomial $\Delta_{K, \rho}(t)$ becomes a polynomial. 
Proof. We first assume that $\rho$ is an irreducible representation. For simplicity, we denote the commutator subgroup $[G(K), G(K)]$ by $G^{\prime}(K)$. Let us consider the following two cases:

(i) $\left.\rho\right|_{G^{\prime}(K)}$ is a nonabelian representation.

(ii) $\left.\rho\right|_{G^{\prime}(K)}$ is an abelian representation.

Case (i). At first, by the assumption, we can choose elements $\gamma, \gamma^{\prime} \in G^{\prime}(K)$ so that

$$
\rho(\gamma) \rho\left(\gamma^{\prime}\right) \neq \rho\left(\gamma^{\prime}\right) \rho(\gamma) \text {. }
$$

We may assume that both of $\rho(\gamma)$ and $\rho\left(\gamma^{\prime}\right)$ have the eigenvalue 1. If not, the assertion follows from Proposition 2.1.

Taking conjugation, we can put

$$
\rho(\gamma)=\left(\begin{array}{cc}
1 & s_{1} \\
0 & 1
\end{array}\right) \quad \text { and } \rho\left(\gamma^{\prime}\right)=\left(\begin{array}{cc}
1 & 0 \\
s_{2} & 1
\end{array}\right)
$$

where $s_{1}, s_{2} \in \mathbb{F}^{\times}=\mathbb{F}-\{0\}$ (see [15] for example). An easy calculation shows that the matrix $\rho\left(\gamma \gamma^{\prime}\right)=\rho(\gamma) \rho\left(\gamma^{\prime}\right)$ does not have the eigenvalue 1. Hence the claim holds in this case.

Case (ii). We suppose that the eigenvalue of $\rho(\gamma)$ is 1 for any $\gamma \in G^{\prime}(K)$. Since the restricted map $\left.\rho\right|_{G^{\prime}(K)}$ is an abelian one, we may assume that the images of $G^{\prime}(K)$ are upper-triangle matrices. The commutator subgroup $G^{\prime}(K)$ is a normal subgroup of $G(K)$, so that it holds

$$
\rho(\xi) \rho(\gamma) \rho(\xi)^{-1}=\left(\begin{array}{ll}
1 & * \\
0 & 1
\end{array}\right)
$$

for any element $\xi \in G(K)$. Hence we see that $\rho(\xi)$ is also an upper-triangle matrix. However this contradicts the irreducibility of the representation $\rho$.

Finally we consider the case where $\rho: G(K) \rightarrow S L(2, \mathbb{F})$ is a reducible nonabelian representation. In this case, we can suppose that the images of generators in a Wirtinger presentation $P(G(K))$ have the following forms:

$$
\rho\left(x_{1}\right)=\left(\begin{array}{cc}
a_{1} & b_{1} \\
0 & a_{1}^{-1}
\end{array}\right), \quad \ldots \quad, \quad \rho\left(x_{u}\right)=\left(\begin{array}{cc}
a_{u} & b_{u} \\
0 & a_{u}^{-1}
\end{array}\right),
$$

where $a_{i} \in \mathbb{F}^{\times}$and $b_{i} \in \mathbb{F}$.

Since $x_{i} x_{j}^{-1}(i \neq j)$ is an element of the commutator subgroup $G^{\prime}(K)$, we see that $a_{i}=a_{j}$ holds for any $i, j$. We then put $a=a_{i}$ for simplicity. Here all matrices are upper-triangle matrices, so that the computation of the determinant does not depend on the off-diagonal entries $b_{1}, \ldots, b_{u}$. Hence it is the same as the one that all matrices are diagonal. Thus the numerator of $\Delta_{K, \rho}(t)$ coincides with the product of the original Alexander polynomials which are evaluated at $a t$ and $a^{-1} t$, that is, $\operatorname{det} M_{j}=\Delta_{K}(a t) \Delta_{K}\left(a^{-1} t\right)$.

On the other hand, the denominator of $\Delta_{K, \rho}(t)$ in this case is just $(t-$ $a)\left(t-a^{-1}\right)$. To finish the proof, we have only to show that two points $t=a$ and $a^{-1}$ are zeros of the numerator. However this follows from the fact that $\rho$ is a representation of $G(K)$ if and only if $a^{2}$ is a zero of $\Delta_{K}(t)$ and $\left(b_{1}, \ldots, b_{u}\right)$ is an eigenvector of the Alexander matrix (see de Rham [2]). In fact, we see that $t=a$ is a zero of $\Delta_{K}(a t)$ and $t=a^{-1}$ is a zero of $\Delta_{K}\left(a^{-1} t\right)$. This completes the proof. 
A knot in the 3-sphere $S^{3}$ is called a fibered knot if its exterior has the structure of a surface bundle over the circle $S^{1}$.

In our previous paper [3], we showed that the twisted Alexander polynomial $\Delta_{K, \rho}(t)$ of a fibered knot $K$ associated to a representation $\rho: G(K) \rightarrow$ $S L(2 n, \mathbb{F})$ is expressed as a rational function of monic polynomials. Here a polynomial

$$
a_{m} t^{m}+\ldots+a_{1} t+a_{0} \in \mathbb{F}[t]
$$

is called a monic polynomial if the coefficient $a_{m}$ is one. It should be noted that the notion of a monic polynomial makes sense for $\Delta_{K, \rho}(t)$, because it is well-defined up to a factor $t^{k}(k \in \mathbb{Z})$. Therefore combining this result with Theorem 3.1, we have more effective assertion for fibered knots.

Theorem 3.2. Let $\rho: G(K) \rightarrow S L(2, \mathbb{F})$ be a nonabelian representation of a genus $g$ fibered knot $K$. Then the twisted Alexander polynomial $\Delta_{K, \rho}(t)$ is a monic polynomial of degree $4 g-2$.

Proof. For a fibered knot $K$ of genus $g$, we can take the following presentation of $G(K)$ coming from the structure of a surface bundle over the circle:

$$
P(G(K))=\left\langle x_{1}, \ldots, x_{2 g}, h \mid r_{i}=h x_{i} h^{-1} \varphi_{*}\left(x_{i}\right)^{-1}, 1 \leq i \leq 2 g\right\rangle,
$$

where $x_{1}, \ldots, x_{2 g}$ is a generating system of the fundamental group of the fiber surface, $h$ is a generator corresponding to the meridian of $K$ and $\varphi_{*}$ denotes the automorphism of the surface group induced by the monodromy $\varphi$.

We then easily see that the degree of $\operatorname{det} M_{2 g+1}$ is equal to $4 g$. Hence the degree of $\Delta_{K, \rho}(t)$ is $4 g-2$, because $\operatorname{det} \Phi(h-1)=t^{2}-(\operatorname{tr} \rho(h)) t+1$. The monicness follows from [3] Theorem 3.1.

Remark 3.3. A similar result on the fibered knots was given by Cha [1]. $\mathrm{He}$ described a necessary condition that a knot in $S^{3}$ was fibered in the framework of Kirk and Livingston [6] (after a slight generalization).

\section{Examples}

Example 4.1. Let $K$ be the trefoil knot $3_{1}$. It is well-known that $G(K)$ allows a presentation

$$
P(G(K))=\left\langle x, y \mid r=x y x y^{-1} x^{-1} y^{-1}\right\rangle .
$$

The abelianization $\alpha: G(K) \rightarrow\langle t\rangle$ is given by $\alpha(x)=\alpha(y)=t$.

Let us consider the trivial representation

$$
\rho_{0}: G(K) \rightarrow S L(2, \mathbb{F})
$$

as an example of abelian representations. An easy calculation shows that the Alexander matrix $M$ is given by

$$
\left(\Phi\left(\frac{\partial r}{\partial x}\right), \Phi\left(\frac{\partial r}{\partial y}\right)\right)=\left(\begin{array}{cccc}
1-t+t^{2} & 0 & -1+t-t^{2} & 0 \\
0 & 1-t+t^{2} & 0 & -1+t-t^{2}
\end{array}\right) .
$$


We also have

$$
\Phi(x-1)=\Phi(y-1)=\left(\begin{array}{cc}
t-1 & 0 \\
0 & t-1
\end{array}\right) .
$$

Therefore the twisted Alexander polynomial of $G(K)$ associated to $\rho_{0}$ is

$$
\Delta_{K, \rho_{0}}(t)=\frac{\left(t^{2}-t+1\right)^{2}}{(t-1)^{2}},
$$

and this is not a polynomial.

Remark 4.2. If $\Gamma$ is a finitely presentable group which is not a knot group, in general, $\Delta_{\Gamma, \rho}(t)$ is not a polynomial even though a representation $\rho$ is nonabelian (see [13] Examples 4.2 and 4.3).

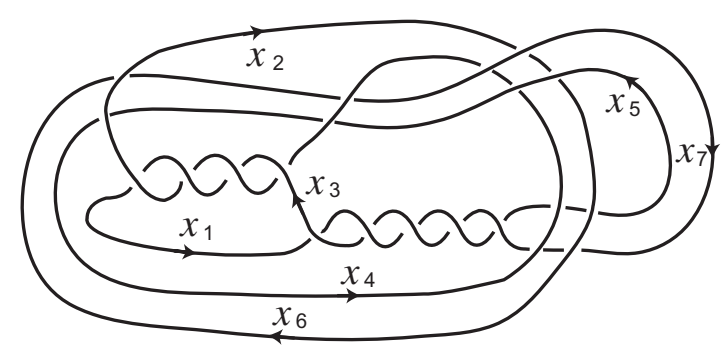

Fig. 1.

Example 4.3. Let $K$ be the knot illustrated in Figure 1. This knot $K$ was first observed in the paper [3]. The Alexander polynomial of $K$ is given by the monic polynomial $t^{4}-t^{3}+t^{2}-t+1$. We also see that $K$ has the genus 2 . Thus we can say nothing on the fiberedness of $K$ from information of the Alexander polynomial.

The knot group $G(K)$ has a presentation with seven generators $x_{1}, \ldots, x_{7}$ and six relations:

$$
\begin{aligned}
& r_{1}: x_{2} x_{1}=x_{3} x_{2} x_{1} x_{2} x_{1}^{-1} x_{2}^{-1}, \\
& r_{2}: x_{6} x_{5} x_{6}^{-1}=x_{4} x_{3} x_{1}^{-1} x_{3} x_{1}^{-1} x_{3} x_{1} x_{3}^{-1} x_{1} x_{3}^{-1} x_{1} x_{3}^{-1} x_{4}^{-1}, \\
& r_{3}: x_{6} x_{7} x_{6}^{-1}=x_{4} x_{3} x_{1}^{-1} x_{3} x_{1}^{-1} x_{3} x_{1} x_{3}^{-1} x_{1} x_{3}^{-1} x_{4}^{-1}, \\
& r_{4}: x_{5} x_{6} x_{5}^{-1}=x_{7} x_{2} x_{7}^{-1} \\
& r_{5}: x_{2} x_{6} x_{2}^{-1}=x_{3} x_{2} x_{1} x_{2} x_{1}^{-1} x_{2}^{-1} x_{3}^{-1} x_{7} x_{3} x_{2} x_{1} x_{2}^{-1} x_{1}^{-1} x_{2}^{-1} x_{3}^{-1}, \\
& r_{6}: x_{5} x_{4} x_{5}^{-1} x_{7}=x_{7} x_{3} x_{2} x_{1} x_{2} x_{1}^{-1} x_{2}^{-1} x_{3}^{-1} .
\end{aligned}
$$

Let $\mathbb{F}_{7}$ be the finite field of cardinality 7 and $\rho: G(K) \rightarrow S L\left(2, \mathbb{F}_{7}\right)$ the nonabelian representation defined as follows:

$$
\begin{aligned}
& \rho\left(x_{1}\right)=\left(\begin{array}{ll}
3 & 3 \\
3 & 1
\end{array}\right), \quad \rho\left(x_{2}\right)=\left(\begin{array}{ll}
5 & 1 \\
1 & 6
\end{array}\right), \quad \rho\left(x_{3}\right)=\left(\begin{array}{ll}
0 & 1 \\
6 & 4
\end{array}\right), \quad \rho\left(x_{4}\right)=\left(\begin{array}{ll}
6 & 4 \\
2 & 5
\end{array}\right), \\
& \rho\left(x_{5}\right)=\left(\begin{array}{ll}
6 & 6 \\
6 & 5
\end{array}\right), \quad \rho\left(x_{6}\right)=\left(\begin{array}{ll}
6 & 1 \\
1 & 5
\end{array}\right) \quad \text { and } \quad \rho\left(x_{7}\right)=\left(\begin{array}{ll}
1 & 2 \\
1 & 3
\end{array}\right) \text {. }
\end{aligned}
$$


A straightforward calculation shows that

$$
\begin{aligned}
\Delta_{K, \rho}(t) & =\frac{t^{6}+2 t^{5}+4 t^{4}+2 t^{3}+4 t^{2}+2 t+1}{t^{2}+3 t+1} \\
& =t^{4}+6 t^{3}+6 t^{2}+6 t+1 .
\end{aligned}
$$

This polynomial is monic but does not have the degree $6=4 \cdot 2-2$. Hence the knot $K$ is not fibered.

Remark 4.4. We have used Kodama's program "KNOT" [10] to find a representation $\rho: G(K) \rightarrow S L\left(2, \mathbb{F}_{7}\right)$ in Example 4.3. The latest version of KNOT can give us a list of linear representations into $S L\left(2, \mathbb{F}_{p}\right)$ and the numerator of the twisted Alexander polynomial associated to such representations.

\section{References}

[1] J. C. CHA, Fibred knots and twisted Alexander invariants, Trans. Amer. Math. Soc. 355 (2003), 4187-4200.

[2] G. DE RHAM, Introduction aux polynomes d'un næud, Enseign. Math. 13 (1968), 187-194.

[3] H. Goda, T. KitANO and T. MoRIFUJi, Reidemeister torsion, twisted Alexander polynomial and fibered knots, Comment. Math. Helv. 80 (2005), 51-61.

[4] H. Goda and T. Morifuji, Twisted Alexander polynomial for $S L(2, \mathbb{C})$-representations and fibered knots, C. R. Math. Acad. Sci. Soc. R. Can. 25 (2003), 97-101.

[5] B. JIANG and S. WANG, Twisted topological invariants associated with representations, In: "Topics in Knot Theory", NATO Adv. Sci. Inst. Ser. C Math. Phys. Sci. 399, Kluwer Academic Publishers, Dordrecht, 1993, 211-227.

[6] P. KIRK and C. Livingston, Twisted Alexander invariants, Reidemeister torsion, and Casson-Gordon invariants, Topology 38 (1999), 635-661.

[7] P. KiRK and C. Livingston, Twisted knot polynomials: inversion, mutation and concordance, Topology 38 (1999), 663-671.

[8] T. Kitano, Twisted Alexander polynomial and Reidemeister torsion, Pacific J. Math. 174 (1996), 431-442.

[9] T. Kitano, M. Suzuki and M. Wada, Twisted Alexander polynomial and surjectivity of a group homomorphism, preprint.

[10] K. KoDAma, http://www.math.kobe-u.ac.jp/HOME/kodama/knot.html

[11] X. S. Lin, Representations of knot groups and twisted Alexander polynomials, Acta Math. Sin. (Engl. Ser.) 17 (2001), 361-380.

[12] T. MoRIfujI, A twisted invariant for finitely presentable groups, Proc. Japan Acad. Ser. A Math. Sci. 76 (2000), 143-145.

[13] T. MorifujI, Twisted Alexander polynomial for the braid group, Bull. Austral. Math. Soc. 64 (2001), 1-13.

[14] L. Neuwirth, "Knot Groups", Annals of Mathematics Studies, No. 56, Princeton University Press, Princeton, N.J., 1965. 
[15] R. RILEY, Nonabelian representations of 2-bridge knot groups, Quarterly J. Math. Oxford (2) 35 (1984), 191-208.

[16] M. SuZUKI, Twisted Alexander polynomial for the Lawrence-Krammer representation, Bull. Austral. Math. Soc. 70 (2004), 67-71.

[17] M. WADA, Twisted Alexander polynomial for finitely presentable groups, Topology 33 (1994), 241-256.

Department of Mathematical and Computing Sciences Tokyo Institute of Technology

2-12-1-W8-43 Oh-okayama, Meguro-ku

Tokyo 152-8552, Japan

kitano@is.titech.ac.jp

Department of Mathematics

Tokyo University of Agriculture

and Technology

2-24-16 Naka-cho, Koganei

Tokyo 184-8588, Japan

morifuji@cc.tuat.ac.jp 